\title{
AN ASYMPTOTIC FORMULA OF GELFAND AND GANGOLLI FOR THE SPECTRUM OF $\boldsymbol{\Gamma} \backslash G$
}

\author{
NOLAN R. WALLACH
}

\section{Introduction}

In [6], Gelfand outlined a proof of an asymptotic formula for the distribution of multiplicities of spherical principal series in $L^{2}(\Gamma \backslash G)$, where $G$ is a connected semi-simple Lie group with finite center and $\Gamma$ is a discrete subgroup of $G$ so that $\Gamma \backslash G$ is compact (see Corollary 1.3 for a formulation of this formula). As pointed out by Gangolli [3] the formula of Gelfand is marginally wrong and the proof of the formula (even in the case $G=S L(2, R)$ ) has a gap. In Gangolli [3] a method using the heat equation was used to prove the (corrected) Gelfand formula for $G$ complex semi-simple. Also Gangolli and Warner have in an as yet unpublished manuscript proved the Gelfand formula if $\Gamma$ has no noncentral elements of finite order. In this paper we use the asymptotic expansion of the fundamental solution of the heat equation to prove a general asymptotic formula which we now describe.

Let $G$ and $\Gamma$ be as above. Let $K$ be a maximal connected compact subgroup of $G$. Let $\hat{G}$ (resp. $\hat{K}$ ) denote the set of equivalence classes of irreducible unitary representations of $G$ (resp. $K$ ). If $\tau \in \hat{K}$, let $d_{\tau}$ be the dimension of any element of the class $\tau$. If $\omega \in \hat{G}$, and $\tau \in \hat{K}$, then let $\left[\tau:\left.\omega\right|_{K}\right]$ denote the multiplicity of $\tau$ in $\omega$ looked at as a direct sum of irreducible representations of $K$ (i.e., $\left.\omega=\sum\left[\tau:\left.\omega\right|_{K}\right] \tau\right)$. If $\omega \in \hat{G}$, let $\lambda_{\omega}$ be the value of the Casimir operator of $G$ on any element of the class $\omega$. Let $Z(G)$ be the center of $G$ and let $Z(\Gamma)$ $=Z(G) \cap \Gamma$. Let $\hat{K}_{\Gamma}$ be the subset of $\hat{K}$ consisting of those $\tau$ such that $Z(\Gamma)$ acts trivially on any element of the class $\tau$. Let $\Pi_{\Gamma}$ denote the right regular representation of $G$ on $L^{2}(\Gamma \backslash G)$. Then $\Pi_{\Gamma}=\sum_{\omega \in \hat{G}} n_{\Gamma}(\omega) \omega, n_{\Gamma}(\omega) \in Z$, $n_{\Gamma}(\omega) \geq 0$. Our main result is

Theorem 1.1. There is a constant $C_{G}$ depending only on $G$ so that if $\tau \in \hat{K}_{\Gamma}$ and if $[Z(\Gamma)]$ is the number of elements in $Z(\Gamma)$, then

$$
\begin{aligned}
\sum_{\omega \in \hat{G}} n_{\Gamma}(\omega)\left[\tau:\left.\omega\right|_{K}\right] e^{t \lambda_{\omega}}= & C_{G} d_{\tau} \frac{[Z(\Gamma)]}{(4 \pi t)^{d / 2}} \operatorname{vol}(\Gamma \backslash G) \\
& +o\left(t^{-d / 2}\right) \quad \text { as } t \rightarrow 0, \quad t>0,
\end{aligned}
$$

where $\operatorname{vol}(\Gamma \backslash G)$ is the volume of $\Gamma \backslash G$ relative to a fixed choice of Haar

Received June 28, 1974. 
measure on $G$, and $d=\operatorname{dim} G / K=\operatorname{dim} G-\operatorname{dim} K$.

It should be pointed out that if $\tau$ is the class of the trivial representation of $K, 1$, then $\left[1:\left.\omega\right|_{K}\right]=0$ or 1 for $\omega \in \hat{G}$.

Using the Gärding inequality we give a simple proof of the following result of Gangolli-Warner [5] (for $\tau=1$ ), Harish-Chandra (unpublished) in general.

Theorem 1.2. If $\tau \in \hat{K}$, then

$$
\sum\left[\tau:\left.\omega\right|_{K}\right] n_{\Gamma}(\omega)\left(1+\left|\lambda_{\omega}\right|\right)^{-d / 2-\varepsilon}<\infty
$$

for all $\varepsilon>0, d=\operatorname{dim}(G / K)$ as before.

Of course, if $\tau \notin \hat{K}_{\Gamma}$ then $\left[\tau:\left.\omega\right|_{K}\right]=0$ when $n_{\Gamma}(\omega) \neq 0$. Hence Theorem 1.2 has interest only in the case $\tau \in \hat{K}_{\Gamma}$.

The above theorem combined with Theorem 1.1 and a Tauberian argument (see Gangolli [3], [4]) implies the Gelfand conjecture for split rank $G$ equal to one. In this case the result has already been proved by Eaton [1].

\section{The equivariant heat equation}

Let $M$ be a compact, connected manifold, and let $G$ be a finite group acting effectively on $M$ by diffeomorphisms (that is, if $g x=x$ for all $x \in M$, then $g$ is the identity element of $G$ ). We include the following well-known result for completeness.

Lemma 2.1. If $g \in G, g \neq e$ ( $e:$ the identity of $G)$ and $M_{g}=\{x \in M \mid g x$ $=x\}$, then $M_{g}$ has measure zero in $M$ (see the proof for the meaning of this).

Proof. Let $\langle$,$\rangle be a Riemannian structure on M$ so that $G$ acts by isometries. Let $p_{0} \in M_{g}$. Let $\operatorname{Exp}_{p_{0}}$ be the exponential map of $(M,<>$ ) (see Helgason [8]), and let $r>0$ be so small that if $B_{p_{0}}(r)=\left\{x \in T(M)_{p_{0}} \mid\langle x, x\rangle\left\langle r^{2}\right\}\right.$, then $\operatorname{Exp}_{p_{0}}: B_{p_{0}}(r) \rightarrow U=\operatorname{Exp}_{p_{0}}\left(B_{p_{0}}(r)\right)$ is a diffeomorphism. If $g \in G-\{e\}$ and $x \in T(M)_{p_{0}}$, then $g \cdot \operatorname{Exp}_{p_{0}}(x)=\operatorname{Exp}_{p_{0}}\left(g_{* p_{0}}(x)\right)\left(g_{* p_{0}}\right.$ is the differential of the action of $g$ at $\left.p_{0}\right)$. Thus, if $\langle x, x\rangle\left\langle r^{2}\right.$ and $g \cdot \operatorname{Exp}_{\rho_{0}}(x)=\operatorname{Exp}_{p_{0}}(x)$, then $g_{* p_{0}}(x)=x$. Now $g_{* p_{0}}$ preserves $\langle$,$\rangle at p_{0}$. Hence, if $V_{p_{0}}=\{x \in$ $\left.T(M)_{p_{0}} \mid g_{* p_{0}} x=x\right\}$, then $T(M)_{p_{0}}=V_{p_{0}} \oplus V_{p_{0}}^{\perp}$ and, by the above, $\operatorname{Exp}_{p_{0}}\left(V_{p_{0}}\right)$ $=U \cap M_{g}$. If $V_{p_{0}}=T(M)_{p_{0}}$, then $g \cdot \operatorname{Exp}_{0}(x)=\operatorname{Exp}_{0}(x)$ for all $X \in T(M)_{p_{0}}$. Since $\operatorname{Exp}_{0}\left(T(M)_{p_{0}}\right)=M, g$ is the identity, and therefore $\operatorname{dim} V_{p_{0}}<\operatorname{dim} T(M)_{p_{0}}$. Thus $\operatorname{Exp}_{p_{0}}\left(V_{p_{0}}\right)$ is a submanifold of $U$ of dimension less than $n$. Hence $U \cap M_{g}$ has measure zero relative to any coordinate system. Since $M_{g}$ can be covered by a finite number of such $U$, the result follows.

Corollary 2.2. Let $\stackrel{\circ}{M}=\{x \in M \mid g x \neq x$ for any $g \neq e\}$. Then $M-\dot{M}$ has measure zero in $M$.

Proof. $M-\stackrel{M}{M}=U_{g \neq e} M_{g}$.

Let $E \stackrel{p}{\longrightarrow} M$ be a $C^{\infty}$ Hermitian $G$-vector bundle over $M$. That is, $E$ is a complex vector bundle over $M$. If $E_{x}=p^{-1}(x)$, then there is $\langle,\rangle_{x}$ an inner product on $E_{x}$ varying smoothly with $x$, and $G$ acts on $E$ by diffeomorphisms 
such that $g E_{x} \subset E_{g \cdot x}$ and $g: E_{x} \rightarrow E_{g \cdot x}$ is a linear isometry of the fibres.

Let $C^{\infty}(M ; E)$ denote the space of $C^{\infty}$ cross-sections of $E$, and let $(g \cdot f)(x)=$ $g f\left(g^{-1} x\right)$ for $g \in G, f \in C^{\infty}(M, E)$. Suppose that there is an elliptic operator $D$ : $C^{\infty}(M ; E) \rightarrow C^{\infty}(M ; E)$ so that the following hold:

(1) $D(g \cdot f)=g \cdot(D f)$.

(2) If $\xi \in T(M)_{x}^{*}$, then $\sigma(D)(\xi)=-\langle\xi \xi\rangle I$,

where $T(M)^{*}$ is the cotangent bundle of $M$, and $\sigma(D)$ is the top order symbol of $D$, and $\langle$,$\rangle is a Riemannian structure on M$.

(3) If $\mu_{0}$ is the Riemannian measure on $M$ corresponding to $\langle$,$\rangle , then$ for $f_{i} \in C^{\infty}(M ; E), i=1,2$, defining $\int_{M}\left\langle f_{1}(x), f_{2}(x)\right\rangle d \mu_{0}(x)=\left(f_{1}, f_{2}\right)$ we assume $\left(D f_{1}, f_{2}\right)=\left(f_{1}, D f_{2}\right)$ and $(D f, f) \geq 0$ for $f \in C^{\infty}(M ; E)$.

Actually results similar to the ones we shall derive are true under very much less stringent conditions than (1), (2), (3).

Let $\tilde{E} \rightarrow \boldsymbol{R} \times M$ be the pull-back bundle $p_{2}^{*} E=\{(t, v) \mid t \in R, v \in E\}$, $I \times p: p_{2}^{*} E \rightarrow \boldsymbol{R} \times M$ the projection, and $L=\partial / \partial t+D$ the evolution operator associated with $D$.

Let $C^{\infty}(M ; E)_{\lambda}=\left\{f \in C^{\infty}(M ; E) \mid D f=\lambda f\right\}$ for $x \in R$. If $C^{\infty}(M ; E)_{\lambda} \neq(0)$, $\lambda \in \boldsymbol{R}$, then $\lambda \geq 0$. Gärding's inequality (see Palais et. al. [10], F. Warner [3] or Greenfield and Wallach [7]) implies

Lemma 2.3. $\sum_{\lambda \neq 0} \operatorname{dim} C^{\infty}(M ; E)_{\lambda} \lambda^{-d / 2-\varepsilon}<\infty$ for all $\varepsilon>0, d=\operatorname{dim} M$. If $\phi, f, g \in C^{\infty}(M ; E)$, then define

$$
\int_{M}(f \hat{\otimes} g)(x, y) \phi(y) d y=\int_{M}\langle g(y), \phi(y)\rangle d \mu_{0}(y) f(x) .
$$

Let $E \hat{\otimes} E \rightarrow M \times M$ be the exterior tensor product of $E$ with itself. If $h \in C^{\infty}(E \hat{\otimes} E)$, then $\int_{M} \mathrm{~h}(x, y) \phi(y) d \mu_{0}(y)$ makes sense for $\phi \in C^{\infty}(E)$.

For $\lambda \in \boldsymbol{R}$ and $\lambda \geq 0$, let $\phi_{\lambda, 1}, \cdots, \phi_{\lambda, n_{\lambda}}$ be an orthonormal basis of $C^{\infty}(M ; E)_{\lambda}\left(\operatorname{dim} C^{\infty}(M ; E)_{\lambda}=n_{\lambda}<\infty\right.$ by the elliptic regularity theorem). Then Lemma 2.3 implies that

$$
\sum_{\lambda} e^{-\lambda t}\left(\sum_{i=1}^{n_{\lambda}} \phi_{\lambda, i}(x) \hat{\otimes} \phi_{\lambda, i}(y)\right)=K(t, x, y)
$$

defines a $C^{\infty}$ cross-section of

$$
\left.P_{2}^{*}(E \hat{\otimes} E)\right|_{(0, \infty) \times M \times M}, \quad\left(P_{2}(t, x, y)=(x, y)\right) .
$$

It is well known and easily proved that if $\phi \in C^{\infty}(M ; E)$, then the unique solution to the Cauchy problem :

(i) $L f=0$,

(ii) $\lim _{\substack{t \rightarrow 0 \\ t>0}} f(t, x)=\phi(x)$

is given by 


$$
f(t, x)=\int_{M} K(t, x, y) \phi(y) d \mu_{0}(y) .
$$

Set $I_{G}^{\infty}(E)$ equal to the space of all $f \in C^{\infty}(M ; E)$ such that $g \cdot f=f$ for $g \in G$. If $\phi \in I_{G}^{\infty}(E)$, then the uniqueness above implies that if $L f=0$ and $\lim _{\substack{t \rightarrow 0 \\ t>0}} f(t, x)=$ $\phi(x)$, then $g \cdot f\left(t, g^{-1} \cdot x\right)=f(t, x)$ for $g \in G$.

Let $C^{\infty}(M ; E)_{\lambda}^{0}=C^{\infty}(M ; E)_{\lambda} \cap I_{G}^{\infty}(E)$. Then we may assume that $\phi_{\lambda, 1}$, $\cdots, \phi_{\lambda, m_{\lambda}}$ form an orthonormal basis of $C^{\infty}(M ; E)_{\lambda}^{0}$. Let

$$
K_{G}(t, x, y)=\sum_{\lambda} e^{-\lambda t} \sum_{i=1}^{m_{\lambda}} \phi_{\lambda, i}(x) \hat{\otimes} \phi_{\lambda, i}(y) .
$$

Let $(g \cdot f)(t, x)=g f\left(t, g^{-1} \cdot x\right)$ for $f \in C^{\infty}(R \times M ; \tilde{E})$ and $g \in G$. Let $I_{G}^{\infty}(\tilde{E})$ be the $f$ in $C^{\infty}((0, \infty) \times M ; \tilde{E})$ such that $g \cdot f=f$ for $g \in G$.

Clearly, if $(K(t) \phi)(x)=\int_{M} K(t, x, y) \phi(y) d y, t>0$, then $K(t): I_{G}^{\infty}(E) \rightarrow I_{G}^{\infty}(\tilde{E})$. If $\left(K_{G}(t) \phi\right)=\int_{M} K_{G}(t, x, y) \phi(y) d y$ for $t>0$, then $K_{G}(t): C^{\infty}(M ; E) \rightarrow I_{G}^{\infty}(\tilde{E})$.

If $v \in E_{x}$ and $w \in E_{y}$, then set $(g \otimes 1)(v \hat{\otimes} w)=g v \hat{\otimes} w,(1 \otimes g)(v \hat{\otimes} w)=$ $v \hat{\otimes} g w .(g \otimes h)(v \hat{\otimes} w)=g v \hat{\otimes} h w, g, h \in G$. Hence $G \times G$ acts on $E \hat{\otimes} E$. Clearly

$$
K_{G}(t, x, y)=\frac{1}{[G]} \sum_{g \in G}(g \otimes 1) K\left(t, g^{-1} x, y\right)
$$

where $[G]$ is the number of elements in $G$.

We also look at $x \rightarrow K(t, x, x)$ and $x \rightarrow K_{G}(t, x, x)$ as a $C^{\infty}$ cross-section of Hom $(E, E)$. Let $I$ be the identity cross-section. The next result is classical, so we will only sketch its proof.

Lemma 2.4. (a) $K(t, x, x)=(4 \pi t)^{-d / 2} I_{x}+O\left(t^{-(d-1) / 2}\right)$ as $t \rightarrow 0, t>0$.

(b) Let $\rho$ be the Riemannian metric corresponding to $\langle$, $\rangle$ on $M$. Then there are constants $C>0, h>0$ so that

$$
\|K(t, x, y)\| \leq C t^{-d / 2} \exp \left(-h \rho(x, y)^{2} / t\right) .
$$

Here the norm is relative to the tensor product Hermitian structure on $E \hat{\otimes} E$.

Proof (outline). Let $\varepsilon>0$ be such that

(a) $\operatorname{Exp}_{p}: B_{p}(\varepsilon) \rightarrow B(p ; \varepsilon)=\{x \in M \mid \rho(x, p)<\varepsilon\}$ is a diffeomorphism for $p \in M$.

(b) $\left.E\right|_{B(p ; \varepsilon)}$ is a trivial bundle for $p \in M$.

Let $p_{1}, \cdots, p_{N} \in M$ be such that if $U_{i}=B\left(p_{i} ; \varepsilon / 2\right), U_{1} \cup \cdots \cup U_{N}=M$. Let $W_{i}=B\left(p_{i} ; \varepsilon\right)$. Let $\left\{x_{1}^{i}, \cdots, x_{d}^{i}\right\}$ be a corresponding system of normal coordinates on $W_{i}$, and $\Psi_{i}=\left(x_{1}^{i}, \cdots, x_{d}^{i}\right)$ the corresponding chart $\left(\Psi_{i}\left(W_{i}\right)=\right.$ $\left.\left\{\left(x_{1}, \cdots, x_{d}\right) \mid \sum x_{i}^{2}<\varepsilon^{2}\right\}\right)$. Let $\Psi_{i}:\left.E\right|_{W_{i}} \rightarrow W_{i} \times C^{m}$ be a vector bundle isomorphism, and let $\phi_{1}, \cdots, \phi_{N}$ be a partition of unity for $M$, supp $\phi_{i} \subset U_{i}$. 
Let $\xi_{i} \in C^{\infty}(M), 0 \leq \xi_{i}(x) \leq 1, x \in M$, supp $\xi_{i} \subset U_{i}, \xi_{i}(x)=1$ for $x \in \operatorname{supp} \phi_{i}$. If $f \in C^{\infty}(M ; E)$, then $F_{i}=\Psi \circ f \circ \Psi_{i}^{-1}: \Psi_{i}\left(W_{i}\right) \rightarrow \Psi_{i}\left(W_{i}\right) \times C^{m}, F_{i}(x)=$ $\left(x, f_{i}(x)\right) . \Psi_{i} \circ D f \circ \Psi_{i}^{-1}=\left(x, D_{i} f_{i}(x)\right)$ where

$$
D_{i}=-\sum a_{k l}^{i} \frac{\partial^{2}}{\partial x_{k} \partial x_{l}}+\sum b_{k}^{i} \frac{\partial}{\partial x_{k}}+C^{i},
$$

where $\left(a_{k l}^{i}(x)\right)$ is a positive definite matrix $b_{k}^{i}, C^{i} \in C^{\infty}\left(\Psi_{i}\left(W_{i}\right), \operatorname{End}\left(C^{n}\right)\right)$. Let $\left(a^{i, k l}(x)\right)=\left(a_{k l}^{i}(x)\right)^{-1}$, and set

$$
Z_{i}(t, x, y)=(4 \pi t)^{-d / 2} \exp \left(-\frac{1}{4 t} \sum_{k, l} a^{i, k, l}(y)\left(x_{k}-y_{k}\right)\left(x_{l}-y_{l}\right)\right)
$$

for $t>0$.

Define for $f \in C^{\infty}(M ; E)$,

$$
(Z(t) f)(x)=\sum_{i=1}^{N} \xi_{i}(x) \Psi_{i}^{-1}\left(x, \int_{V_{i}} \phi_{i}(y) Z_{i}\left(t, \Psi_{i}(x), \Psi_{i}(y)\right) f_{i}(y) d \mu_{0}(y)\right) .
$$

Then it is easily seen (see Friedman [2, Theorem 1, p. 4]) that

$$
\lim _{\substack{t \rightarrow 0 \\ t>0}}(Z(t) f)(x)=f(x)
$$

for $x \in M$. It is also clear that $Z(t)$ has a $C^{\infty}$ kernel $Z(t, x, y)$. That is, $(Z(t) f)(x)=\int_{M} Z(t, x, y) f(y) d \mu_{0}(y)$ where $Z(t, x, y) \in E_{x} \hat{\otimes} E_{y}$.

If $f \in C^{\infty}((0, \infty) \times M ; \tilde{E}), g \in C^{\infty}(M ; E)$ define $L(f \hat{\otimes} g)=L f \hat{\otimes} g$. Arguing as in Friedman [2, Chapter 1, §4] we define

$$
\Phi_{1}(t, x, y)=-L Z(t, x, y) .
$$

Supposing that $\Phi_{\nu}$ has been defined, set

$$
\Phi_{\nu+1}(t, x, y)=-\int_{0}^{t} \int_{M} L Z(t \sigma, x, \xi) \Phi_{\nu}(\sigma, \xi, y) d \mu_{0}(\xi) d \sigma
$$

Then the above arguments of Friedman imply that if $\Phi(t, x, y)=\sum_{\nu=1}^{\infty} \Phi_{\nu}(t, x, y)$, then $\Phi$ converges uniformly and absolutely on compact subsets of $(0, \infty) \times$ $M \times M$ to a $C^{\infty}$ cross-section of $C^{\infty}\left((0, \infty) \times M \times M ; P_{2}^{*}(E \hat{\otimes} E)\right)$. Furthermore we have that there are $C>0, h>0$ so that

(a) $\|Z(t, x, y)\| \leq C t^{-d / 2} \exp \left(-\frac{h}{t} \rho(x, y)^{2}\right)$,

(b) $\|\Phi(t, x, y)\| \leq C t^{-(d+1) / 2} \exp \left(-\frac{h}{t} \rho(x, y)^{2}\right)$, 
(c) $\|L Z(t, x, y)\| \leq C t^{-(d+1) / 2} \exp \left(-\frac{h}{t} \rho(x, y)^{2}\right)$

for $0<t \leq T<\infty, x, y \in M$.

Also arguing as in [2, Theorem 8, p. 19] we see

$$
K(t, x, y)=Z(t, x, y)+\int_{0}^{t} \int_{M} Z(t-\sigma, x, \xi) \Phi(\sigma, \xi, y) d \mu_{0}(\xi) d \sigma .
$$

Using [2, Lemma 3, p. 15] we see that if

$$
V(t, x, y)=\int_{0}^{t} \int_{M} Z(t-\sigma, x, \xi) \Phi(\sigma, \xi, y) d \mu_{0}(\xi) d \sigma,
$$

then

$$
\|V(t, x, y)\| \leq C t^{-(d+1) / 2} \exp \left(-\frac{h}{t} \rho(x, y)^{2}\right)
$$

for $0<t \leq T$.

The lemma now follows from the fact that $Z(t, x, y)$ obviously satisfies (1), (2) of the lemma.

Lemma 2.5. Let for $\lambda \in R, m_{\lambda}=\operatorname{dim} C^{\infty}(M ; E)_{\lambda}^{0}=\operatorname{dim}\left\{f \in C^{\infty}(M ; E) \mid\right.$ $D f=\lambda f, g \cdot f=f$ for all $g \in G\}$. Let $\operatorname{vol}(M)=\int_{M} d \mu_{0}(x)$. Let $m$ be the fibre dimension of $E$. If $d=\operatorname{dim} M$, then

$$
\sum_{\lambda} m_{\lambda} e^{-\lambda t}=\frac{m}{[G]} \frac{\operatorname{vol}(M)}{(4 \pi t)^{d / 2}}+o\left(t^{-d / 2}\right)
$$

as $t \rightarrow 0, t>0$.

Proof. If $f, g \in C^{\infty}(M ; E)$, define $\operatorname{tr}(f(x) \otimes g(x))=\langle f(x), g(x)\rangle$. Then clearly

$$
\sum_{\lambda} m_{\lambda} e^{-\lambda t}=\int_{M} \operatorname{tr}\left(K_{G}(t, x, x)\right) d \mu_{0}(x)
$$

Now

$$
K_{G}(t, x, y)=\frac{1}{[G]} K(t, x, y)+\frac{1}{[G]} \sum_{g \neq e}(g \otimes 1) \cdot K\left(t, g^{-1} \cdot x, y\right) .
$$

Thus Lemma 2.4 will imply the lemma if we can show that if $g \neq e$ then

$$
\int_{M}\left\|(g \otimes 1) K\left(t, g^{-1} x, x\right)\right\| d \mu_{0}(x)=o\left(t^{-d / 2}\right)
$$

as $t \rightarrow 0, t>0$. 
Let now $g \in G-\{e\}$ be fixed and $\varepsilon>0$ be given. Let $U$ be open in $M$ so that $U \supset M_{g^{-1}}$ (see Lemma 2.1) and $\int_{U} d \mu_{0}(x)<\frac{1}{2} \varepsilon C V, C$ and $V$ to be determined. Let

$$
J(t)=\int_{M}\left\|(g \otimes 1) K\left(t, g^{-1} x, x\right)\right\| d \mu_{0}(x)=\int_{M}\left\|K\left(t, g^{-1} x, x\right)\right\| d \mu_{0}(x) .
$$

Then

$$
J(t)=\int_{M-U}\left\|K\left(t, g^{-1} x, x\right)\right\| d \mu_{0}(x)+\int_{U}\left\|K\left(t, g^{-1} x, x\right)\right\| d \mu_{0}(x) .
$$

Now

$$
\begin{gathered}
\left\|K\left(t, g^{-1} x, x\right)\right\| \leq C t^{-d / 2} \exp \left(-\frac{h}{t} \rho\left(g^{-1} x, x\right)\right) \leq C t^{-d / 2} V, \\
V=\max _{\substack{x, y \in M \\
t \leq 1}} \exp \left(-\frac{h}{t} \rho(x, y)\right) .
\end{gathered}
$$

Thus

$$
t^{d / 2} J(t) \leq \int_{M-U}\left\|K\left(t, g^{-1} x, x\right)\right\| d \mu_{0}(x)+\frac{1}{2} \varepsilon .
$$

Now $M-U$ is compact and $M-U \subset M-M_{g^{-1}}$. Hence there is $\delta>0$ so that if $x \in M-U$ then $\rho\left(g^{-1} x, x\right) \geq \delta$. Applying Lemma 2.4 again we find that $t^{d / 2} J(t) \leq \frac{1}{2} \varepsilon+C \operatorname{vol}(M) e^{-\dot{\delta}^{2} h / t}$ if $t \leq 1$. Take $\mu>0$ so that $e^{-\tilde{\delta}^{2} h / t}<$ $\frac{1}{2} \varepsilon C \operatorname{vol}(M)$ if $0<t<\mu$. Then $t^{d / 2} J(t)<\varepsilon$ for $0<t<\mu$. q.e.d.

In the next section we apply these results to $\Gamma \backslash G$.

\section{Applications to $\Gamma \backslash G$}

Let $G$ be a semi-simple Lie group with finite center and such that $G$ has no connected, compact, normal subgroups. Let $K \subset G$ be a maximal connected, compact subgroup. Let $X=G / K$. Let $g$ be the Lie algebra of $G$, and $B$ the Killing form of $\mathfrak{g}$. Let $\mathfrak{f} \subset \mathfrak{g}$ be the Lie algebra of $K$, and $\mathfrak{p}$ the orthogonal compliment to $\mathfrak{f}$ in relative to $B$. Then it is well known that $\left.B\right|_{\mathfrak{p} \times \mathfrak{p}}$ is positive definite. We put the $G$-invariant Riemannian structure $\langle$,$\rangle on X$; this corresponds to making $\Pi_{*_{e}}: \mathfrak{p} \rightarrow T(X)_{e k}(\Pi: G \rightarrow G / K$ is the natural map, and $\Pi_{*_{e}}$ is its differential at $\left.e \in G\right)$ an isometry of $\left.B\right|_{\mathfrak{p} \times \mathfrak{p}}$ and $\langle,\rangle_{e k}$.

Let now $(\tau, V)$ be an irreducible unitary representation of $K$. We form the $G$-hermitian vector bundle over $X, G \underset{\tau \otimes I}{\times}\left(V \otimes V^{*}\right)=V$ where $G \underset{\tau \otimes I}{\times}\left(V \otimes V^{*}\right)$ is the associated bundle to the principal bundle $K \rightarrow G \stackrel{\Pi}{\longrightarrow} X$ (cf. KobayashiNomizu [9] or Wallach [12]). Then $\boldsymbol{V}$ is completely described as follows : 
(1) If $g$ is in $G$, then $g$ induces a linear map $V_{x} \rightarrow V_{g x}$ which we denote $v \rightarrow g \cdot v$. The corresponding action of $G$ on $V$ is $C^{\infty}$.

(2) The representation of $K$ on $V_{e k}$ given by $v \rightarrow k \cdot v, v \in V_{e k}$, is equivalent to $\left(\tau \otimes I, V \otimes V^{*}\right)$ as a unitary representation.

If $f \in C^{\infty}(X ; V)$, let $(g \cdot f)(x)=g f\left(g^{-1} \cdot x\right)$. Then $g \cdot f \in C^{\infty}(X ; V)$ for $f \epsilon$ $C^{\infty}(X ; V)$. Let $X_{1}, \cdots, X_{n}$ be a basis of $\mathrm{g}$, and let $Y_{1}, \cdots, Y_{n}$ be such that $B\left(X_{i}, Y_{j}\right)=\delta_{i j}$. Then defining $(X \cdot f)(x)=\frac{d}{d t}\left(\left.\exp t X \cdot f(\exp (-t X) \cdot x)\right|_{t=0}\right.$ for $X \in \mathfrak{g}$ and $f \in C^{\infty}(X ; V)$ we set

$$
\Omega_{V} f=\sum_{i=1}^{n} X_{i} Y_{i} \cdot f
$$

Thus $\Omega_{V} g \cdot f=g \Omega_{V} f, g \in G$.

A simple computation shows that if $\xi \in T(X){ }_{e k}$, then $\sigma\left(\Omega_{V}\right)(\xi)=\langle\xi, \xi\rangle I$. Define a $G$-invariant connection on $V$ by $\left(\nabla_{u} f\right)(e k)=(X \cdot f)(e k)$ for $u \epsilon$ $T(G / K)_{e k}, u=\Pi_{*_{e}}(X), X \in \mathfrak{p}$. The corresponding connection on $V$ satisfies

$$
X \cdot\langle\Psi, \eta\rangle=\left\langle\nabla_{X} \Psi, \eta\right\rangle+\left\langle\Psi, \nabla_{X} \eta\right\rangle
$$

Let $\nabla^{2}$ be the connection Laplacian on $V$ corresponding to the connection $V$ and the Riemannian structure on $X$.

Lemma 3.1. Let $\Omega_{K}=-\sum Y_{i}^{2}$ where $Y_{1}, \cdots, Y_{k}$ form a basis of $\mathfrak{t}$ so that $B\left(Y_{i}, Y_{j}\right)=-\delta_{i j}$. Let $\lambda_{\tau}$ be defined by $\tau\left(\Omega_{K}\right)=\lambda_{\tau} I$ (Schur's lemma implies this makes sense). If $f \in C^{\infty}(X ; V)$, then

$$
\Omega_{V} f=\nabla^{2} f+\lambda_{\tau} f .
$$

Proof. If $f \in C^{\infty}(X ; \boldsymbol{V})$, define $\tilde{f}(g)=g^{-1} \cdot f(g k)$. Then $\tilde{f}: G \rightarrow V_{e k}$ and $\tilde{f}(g k)=k^{-1} \tilde{f}(g)$ for $k \in K, g \in G$. Let $\left(L_{g} \phi\right)(x)=\phi\left(g^{-1} x\right)$ for $\phi: G \rightarrow V_{e k}$, where $\phi$ is of class $C^{\infty}$, and $g, x \in G$. We note that if $A(f)=\tilde{f}$ for $f \in C^{\infty}(X ; V)$ and we define $B(\phi)(g k)=g \cdot \phi(g)$ for $\phi: G \rightarrow V_{e k}$, then $\phi(g k)=k^{-1} \cdot \phi(g)$, $k \in K, g \in G$. Thus $B(\phi) \in C^{\infty}(X ; V)$ and $A B(\phi)=\phi, B A(f)=f$.

Let $\left(R_{X} \phi\right)(g)=\left.\frac{d}{d t} \phi($ gexpt $X)\right|_{t=0}$ for $X \in \mathrm{g}$ and $\phi: G \rightarrow V_{e k}, \phi$ being of class $C^{\infty}$. Then a direct computation shows that if $X_{1}, \cdots, X_{p}$ form an orthonormal basis of $\mathfrak{p}$ relative to $\left.B\right|_{\mathfrak{p} \times \mathfrak{p}}$, then $A\left(\nabla^{2} f\right)=\sum_{i=1}^{p} R_{X_{i}}^{2} A(f)$. Also

$$
\begin{aligned}
A\left(\Omega_{V} f\right) & =\sum_{i=1}^{p} R_{X_{i}}^{2} A(f)-\sum_{i=1}^{p} R_{X_{i}}^{2} A(f) \\
& =\sum_{i=1}^{p} R_{X_{i}}^{2} A(f)+\tau\left(\Omega_{K}\right)(A(f))=A\left(V^{2} f\right)+\lambda_{\tau} A(f) .
\end{aligned}
$$

Applying $B$ gives the result. 
Let now $\Gamma \subset G$ be a discrete subgroup so that $\Gamma \backslash G$ is compact and $g \Gamma g^{-1} \cap K=\{e\}$ for all $g \in G$. Then $\Gamma$ acts freely and properly discontinuously on $X$ and $V$. We may thus form $E=\Gamma \backslash V \rightarrow \Gamma \backslash X=M$.

Since $\Gamma$ acts by isometries on $X$, we may "push" the Riemannian structure and volume element on $X$ down to $\mathrm{M}$. The Hermitian structure on $V$ induces a Hermitian structure on $E$. Finally $\Omega_{V}$ and $\nabla^{2}$ are $G$-invariant operators on $V$, and thus the induced second order elliptic operators on E. We still have $\Omega_{V}=\nabla^{2}+\lambda_{\tau} I$.

Set $D=-\left(\Omega_{V}-\lambda_{\tau} I\right)=-\nabla^{2}$. Then $(D f, f) \geq 0, D=D^{*}$ and $\sigma(D, \xi)=$ $-\langle\xi, \xi\rangle$. Thus $D$ satisfies (1), (2), (3) of $\S 2$.

Let $\boldsymbol{f}(g)(k)=f(g k)$ for $f \in C^{\infty}(\Gamma \backslash G)$. Then $f: \Gamma \backslash G \rightarrow C^{\infty}(K)$. Let $C_{\tau}^{\infty}(K)$ be the subspace of $C^{\infty}(K)$ spanned by the matrix entries of $(\tau, V)$. Let $\chi_{\tau}$ be the character of $(\tau, V)$. Define $f_{\tau}(g)=\int_{K} \chi_{\tau}(e) \overline{\chi_{\tau}(k)} f(g k) d k$ for $f \in C^{\infty}(\Gamma \backslash G)$. Then $f_{\tau}: \Gamma \backslash G \rightarrow C_{\tau}^{\infty}(K)$ and $\boldsymbol{f}_{\tau}(g u)(k)=\boldsymbol{f}_{\tau}(g)(u k)$. Let $C_{\tau}^{\infty}(\Gamma \backslash G)=\{f \epsilon$ $\left.C^{\infty}(\Gamma \backslash G) \mid f_{\tau}=f\right\}$. Let $(\mu(k) \phi)(x)=\phi\left(k^{-1} x\right)$ for $\phi \in C_{\tau}^{\infty}(K)$, and $k, x \in K$. We therefore see that if $f \in C_{\tau}^{\infty}(\Gamma \backslash G)$, then $f: \Gamma \backslash G \rightarrow C_{\tau}^{\infty}(K)$ and $f(g u)=\mu(u)^{-1} f(x)$ for $x, u \in K$.

Let $\Pi_{\Gamma}$ be the right regular representation of $G$ on $L^{2}(\Gamma \backslash G)$. That is, if $\phi \in L^{2}(\Gamma \backslash G)$ then $\left(\pi_{\Gamma}(x) \phi\right)(\Gamma g)=\phi(\Gamma g x)$ for $g, x \in G$. Then it is well known that $\pi_{\Gamma}=\sum_{\omega \in G} n_{\Gamma}(\omega) \omega$. $\hat{G}$ is the set of all equivalence classes of irreducible unitary representations of $G$.

If $\lambda \in R$, let $\hat{G}_{\lambda}=\left\{\omega \in G \mid \pi_{\omega}(\Omega)=-\lambda I\right.$ for every $\pi_{\omega}$ in the class $\left.\omega\right\}$.

Lemma 3.2. Set $C^{\infty}(M ; E)_{\lambda}=\left\{\phi \in C^{\infty}(M ; E) \mid D \phi=\lambda \phi\right\}$. Then

$$
\operatorname{dim} C^{\infty}(M ; E)_{\lambda}=\sum_{\omega \in \hat{G}_{\lambda-\lambda_{\tau}}} n_{\Gamma}(\omega) \cdot\left[\tau:\left.\omega\right|_{K}\right] d_{\tau},
$$

$d_{\tau}=\operatorname{dim} V=\chi_{\tau}(e)$.

Proof. $E$ can be looked upon as the set of equivalence classes of pairs $(x, v), x \in \Gamma \backslash G, v \in V \otimes V^{*}$ with $\left(x k,(\tau(k) \otimes I)^{-1} v\right) \equiv(x, v)$ for $k \in K$. Let $[x, v]$ denote the equivalence class of $(x, v)$. Let $C^{\infty}(\Gamma \backslash G ; \tau)$ denote the space of all $\phi: \Gamma \backslash G \rightarrow V \otimes V^{*}, \phi \in C^{\infty}$ and $\phi(x k)=\left(\tau(k)^{-1} \otimes I\right) \phi(x)$. Define $B(\phi)(x)$ $=[x, \phi(x)]$ for $\phi \in C^{\infty}(\Gamma \backslash G ; \tau)$. Then $B$ defines a bijection of $C^{\infty}(\Gamma \backslash G ; \tau)$ and $C^{\infty}(M ; E)$. Now as a representation of $K,\left(\mu, C_{\tau}^{\infty}(K)\right)$ is equivalent to $\left(\tau \otimes I, V \otimes V^{*}\right)$. Thus we have $B^{-1}: C^{\infty}(M ; E) \rightarrow C_{\tau}^{\infty}(\Gamma \backslash G) . B^{-1}$ is bijective and extends to a bounded bijective operator on the appropriate $L^{2}$-completions. But then $B^{-1}\left(C_{\tau}^{\infty}(M ; E)_{\lambda}\right)=\left\{f \in C_{\tau}^{\infty}(\Gamma \backslash G) \mid \Omega f=-\left(\lambda-\lambda_{\tau}\right) f\right\}$. If $f \in C_{\tau}^{\infty}(\Gamma \backslash G)$, then $f=\sum f_{\omega}, f_{\omega} \in n_{\Gamma}(\omega) H_{\omega},\left(\pi_{\omega}, H_{\omega}\right) \in \omega$. Thus $\Omega f=\sum \lambda_{\omega} f_{\omega}$, and the result now follows.

Suppose now that $\Gamma_{1} \subset G$ is an arbitrary discrete subgroup so that $\Gamma_{1} \backslash G$ is compact. Then there is a normal subgroup $\Gamma$ of $\Gamma_{1}$ so that $\Gamma$ acts freely and properly discontinuously on $X$, and if $H=\Gamma_{1} \backslash \Gamma$ then $H$ is a finite group of isometries of $\Gamma \backslash X$ (cf. Raghunathan [11]). 
Now $E \rightarrow M=\Gamma \backslash X$ is an $H$-vector bundle, since $E$ is the associated bundle to $\Gamma \backslash G \rightarrow \Gamma \backslash X$ and $H$ acts on the left on $\Gamma \backslash G$. Let $Z\left(\Gamma_{1}\right)=\Gamma_{1} \cap Z(G)$, where $Z(G)$ is the center of $G$. We note that since $Z(G) \subset K, Z\left(\Gamma_{1}\right) \subset K$. Also, if $z \in Z(G)$ then $\tau(z)=\xi_{\tau}(z) I, \xi_{\tau}: Z(G) \rightarrow T^{1}$ being a character. Thus, if $\gamma \in Z\left(\Gamma_{1}\right)$ and $h=\gamma \Gamma$, then $h \cdot v=\xi_{z}(\gamma) v$ for $v \in E$. We therefore see that $C^{\infty}(M ; E)_{\lambda}^{0}=\left\{f \in C^{\infty}(M ; E)_{\lambda} \mid h \cdot f=f, h \in H\right\} \neq 0$ only if $\left.\tau\right|_{Z_{\left(\Gamma_{1}\right)}}=I$.

We assume that $\left.\tau\right|_{Z\left(\Gamma_{1}\right)}=I$. Arguing as above we find

Lemma 3.3. $\operatorname{dim} C^{\infty}(M ; E)_{\lambda}^{0}=\sum_{\omega \in \hat{G}_{\lambda-\lambda_{\tau}}} n_{\Gamma_{1}}(\omega)\left[\tau:\left.\omega\right|_{K}\right] d_{\tau}$, where $\Pi_{\Gamma_{1}}=$ $\sum n_{\Gamma_{1}}(\omega) \omega$, and $\Pi_{\Gamma_{1}}$ is the right regular representation of $G$ on $L^{2}\left(\Gamma_{1} \backslash G\right)$.

Now $H$ does not necessarily act effectively on $\Gamma \backslash X$. Let $H_{0}=\{h \in H \mid h \Gamma x=$ $\Gamma x$ for all $x \in X\}$. Then, as is easily seen, $H_{0}$ is the image of $Z\left(\Gamma_{1}\right)$ in $H$. Since $Z\left(\Gamma_{1}\right) \cap \Gamma=(e)$, we see that $\left[H_{0}\right]=\left[Z\left(\Gamma_{1}\right)\right]$. Finally $E$ is an $H / H_{0}$ vector bundle if and only if $H_{0}$ acts trivially on the fibres of $E$, that is, if and only if $\tau \in \hat{K}_{\Gamma_{1}}$ (see the introduction for the definition of $\hat{K}_{\Gamma_{1}}$ ).

Combining the above observations with Lemma 3.3 and Lemma 2.5 we see

$$
\begin{aligned}
e^{\lambda_{\tau} t} \sum_{\omega \in \hat{G}} e^{\lambda_{\omega} t} n_{\Gamma_{1}}(\omega) d_{\tau}\left[\tau:\left.\omega\right|_{K}\right]= & \frac{\left[Z\left(\Gamma_{1}\right)\right]}{\left[\Gamma_{1} \backslash \Gamma\right]} t^{-d / 2} \operatorname{vol}(M) d_{\tau}^{2} \\
& +o\left(t^{-d / 2}\right) \quad \text { as } t \rightarrow 0, \quad t>0 .
\end{aligned}
$$

Normalize Haar measure $d g$ on $G$ so that if $X_{1}, \cdots, X_{n}$ form a basis of $\mathrm{g}$ so that $-B\left(X_{i}, \theta X_{j}\right)=\delta_{i j}\left(\left.\theta\right|_{\mathfrak{t}}=I,\left.\theta\right|_{\mathfrak{p}}=-I\right)$, then $d g\left(X_{1}, \cdots, X_{n}\right)=1$. Let $C_{G}^{-1}$ be the volume of $K$ relative to the Riemannian volume element on $K$ corresponding to the inner product $-\left.B\right|_{\mathfrak{t} \times \mathfrak{t}}$. Then

$$
\operatorname{vol}\left(\Gamma_{1} \backslash G\right)=\left[\Gamma_{1} / \Gamma\right]^{-1} \cdot \operatorname{vol}(\Gamma \backslash G)=\left[\Gamma_{1} / \Gamma\right]^{-1} C_{G}^{-1} \operatorname{vol}(\Gamma \backslash X) .
$$

Hence $C_{G}$ vol $\left(\Gamma_{1} \backslash G\right)=\left[\Gamma_{1} / \Gamma\right]^{-1} \cdot \operatorname{vol}(\Gamma \backslash X)$. These observations combined with (1) above prove

Theorem 3.4. There is a constant $C_{G}$ depending only on $G$ so that if $\Gamma$ is a discrete subgroup of $G$ with $\Gamma \backslash G$ compact and if $\tau \in \hat{K}_{\Gamma}$, then

$$
\begin{aligned}
& \sum_{\omega \in \hat{G}} n_{\Gamma}(\omega)\left[\pi:\left.\omega\right|_{K}\right] e^{t \lambda \omega}=C_{G} d_{\tau} \frac{[Z(\Gamma)]}{(4 \pi t)^{d / 2}} \operatorname{vol}(\Gamma \backslash G)+o\left(t^{-d / 2}\right), \\
& \text { as } t \rightarrow 0, \quad t>0 .
\end{aligned}
$$

We also note that Lemma 2.3 combined with Lemmas 3.2 and 3.3 immediately imply Theorem 1.2 of the introduction.

\section{References}

[ 1 ] T. Eaton, Thesis, University of Washington, Seattle, 1973.

[2] A. Friedman, Partial differential equations of parabolic type, Prentice-Hall, Englewood Cliffs, New Jersey, 1964. 
[ 3 ] R. Gangolli, Asymptotic behavior of spectra of compact quotients of certain symmetric spaces, Acta. Math. 121 (1968) 151-192.

[ 4 ] - Spectra of discrete subgroups, Proc. Sympos. Pure Math. Vol. 26, Amer. Math. Soc., 1973, 431-436.

[ 5 ] R. Gangolli \& G. Warner, On Selberg's trace formula, Japan. J. Math., to appear.

[6] I. M. Gelfand, Automorphic forms and the theory of representations, Proc. Internal. Conf. Math. (Stockholm, 1962), Inst. Mittag-Leffler, Djursholm, 1963, 74-85.

[ 7 ] S. Greenfield \& N. R. Wallach, Remarks on global hypoellipticity, Trans. Amer. Math. Soc. 183 (1973) 153-164.

[ 8 ] S. Helgason, Differential geometry and symmetric spaces, Academic Press, New York, 1962.

[9] S. Kobayashi \& K. Nomizu, Foundations of differential geometry. I, Interscience, New York, 1962.

[10] R. Palais et al., Seminar on the Atiyah-Singer index theorem, Annals of Math. Studies, No. 57, Princeton University Press, Princeton, 1965.

[11] M. Raghunathan, Discrete subgroups of Lie groups, Springer, Berlin, 1972.

[12] N. Wallach, Harmonic analysis on homogeneous spaces, Marcel Dekker, New York, 1973.

[13] F. Warner, Foundations of differential geometry and Lie groups, Scott, Foresman and Co., Glenview, Illinois, 1971.

RUTGERS UNIVERSITY 
